\title{
Influence of oxygen vacancies on the electronic structure of $\mathrm{HfO}_{2}$ films
}

\author{
Deok-Yong Cho, ${ }^{1}$ Jae-Min Lee, ${ }^{1}$ S.-J. Oh,,${ }^{1, *}$ Hoyoung Jang, ${ }^{2}$ J.-Y. Kim, ${ }^{3}$ J.-H. Park, ${ }^{2,3}$ and A. Tanaka ${ }^{4}$ \\ ${ }^{1}$ CSCMR and Department of Physics and Astronomy, Seoul National University, Seoul 151-742, Korea \\ ${ }^{2}$ eSSC and Department of Physics, Pohang University of Science and Technology, Pohang 790-784, Korea \\ ${ }^{3}$ Pohang Accelerator Laboratory, Pohang University of Science and Technology, Pohang 790-784, Korea \\ ${ }^{4}$ Department of Quantum Matter, ADSM, Hiroshima University, Higashi-Hiroshima 739-8526, Japan
}

(Received 6 April 2007; revised manuscript received 12 June 2007; published 9 October 2007)

\begin{abstract}
We investigated the unoccupied part of the electronic structure of the oxygen-deficient hafnium oxide $\left(\mathrm{HfO}_{\sim 1.8}\right)$ using soft x-ray absorption spectroscopy at $\mathrm{O} K$ and $\mathrm{Hf} N_{3}$ edges. Band-tail states beneath the unoccupied Hf $5 d$ band are observed in the $\mathrm{O} K$-edge spectra. Combined with ultraviolet photoemission spectrum, this indicates the non-negligible occupation of the Hf $5 d$ state. However, Hf $N_{3}$-edge magnetic circular dichroism spectrum reveals the absence of a long-range ferromagnetic spin order in the oxide. Thus, the small amount of $d$ electron gained by the vacancy formation does not show intersite correlation, contrary to a recent paper [M. Venkatesan et al., Nature 430, 630 (2004)].
\end{abstract}

DOI: 10.1103/PhysRevB.76.165411

PACS number(s): 73.20.Hb, 78.70.Dm, 73.61.Ng, 75.70.-i

\section{INTRODUCTION}

An oxygen vacancy in a large band-gap oxide changes the electrical characteristics by forming defect states inside the band gap. Depending on the position and density of the defect energy levels, the chemical potential of the oxidefrequently identified with the Fermi level $\left(E_{F}\right)$-changes as in the case of doping with other atomic species. Hence, the characterization of the defect level is a prerequisite for understanding the electron transfer across the oxidesemiconductor interface, because the energy barrier height at the interface depends on $E_{F}$ of the oxide. ${ }^{1,2}$ With regard to hafnium oxide, the energy position of this defect level has been estimated theoretically ${ }^{3-5}$ and experimentally ${ }^{6,7}$ to be above the middle of the band gap, so $E_{F}$ is shifted upward in the presence of oxygen vacancies. Since $E_{F}$ is determined from the charge neutrality condition, it is largely influenced by the detailed density of states (DOS) near the band gap; ${ }^{1}$ in this case, the intensity of the features near the conduction band $(\mathrm{CB})$ is expected to be higher than those near the valence band $(\mathrm{VB})$.

Recent density-functional-theoretical (DFT) studies ${ }^{3-5,8-10}$ have revealed that the defect state is the localized $\mathrm{Hf} d$ state. The nonzero occupation in the Hf $d$ shell is very natural, because the Hf atom no longer donates electrons to the adjacent vacant sites. If an intersite correlation existed between the $d$ electrons, even a long-range correlation in the electronic configuration of each site might emerge. Surprisingly, room-temperature long-range ferromagnetism (FM) was recently reported in an oxygen-deficient $\mathrm{Hf}$ oxide film, ${ }^{11-13}$ although an experimental counterevidence shows the absence of FM. ${ }^{14}$ Since the measured magnetization is the macroscopic quantity, it might significantly depend on the purity of the sample. Furthermore, recent DFT studies have revealed that FM can be induced by a hafnium vacancy rather than an oxygen vacancy, while the oxygen vacancy can at the most yield paramagnetism. ${ }^{15-17}$ Thus, it is important to investigate the possible long-range spin order in the oxygen-deficient $\mathrm{HfO}_{x<2}$ from a microscopic point of view, in that the microscopic quantities such as the spin or angular momentum drive the macroscopic properties, i.e., the magnetization. Since these microscopic values are determined from the electronic structure, the study on the local electron configurations of each atomic species will provide the information that is not influenced by a macroscopic deformation (domain interaction, dislocation, etc.) or an intervention of impurities.

Therefore, in this paper, we focus on electron redistribution and the possible long-range spin order in the presence of an oxygen vacancy in $\mathrm{Hf}$ oxide by studying its electronic structure. To investigate the unoccupied electronic structure of the Hf $d$ states, we performed an X-ray absorption spectroscopy (XAS) study on the $\mathrm{HfO}_{x<2}$ film. Since XAS reflects a transition probability from a spatially localized core level to the unoccupied levels, it is sensitive to the unoccupied states originating from the atoms near the photonabsorbing atom. Further, owing to the "locality" that allows transitions irrespective of momentum transfer, it reflects the momentum-averaged electronic structure that would be equivalent to the partial density of states (PDOS) in the absence of an electron correlation. We can choose the photon absorber by tuning the photon energy range to any of the core-level energies of each atomic species. Using the photon energy range $h \nu \sim 530 \mathrm{eV}$, we can measure $\mathrm{O} K$-edge ( $1 \mathrm{~s}$ $\rightarrow 2 p$ ) absorption to investigate the O $2 p$ PDOS. Since parts of $\mathrm{O} 2 p$ wave functions are hybridized with $\mathrm{Hf} 5 d$ wave functions, $\mathrm{O} K$-edge XAS also shows the unoccupied $\mathrm{Hf} 5 d$ energy levels that are hybridized with the $\mathrm{O}$ wave functions. This implies that an $\mathrm{Hf} d$-electron state that stays near the vacant site is hardly observed in O $K$-edge XAS. The situation is described in Fig. 1. The dashed circle near an oxygen atom roughly indicates the probing area of $\mathrm{O} K$-edge XAS. However, at the $\mathrm{Hf} N_{3}$ edge $\left(4 p_{3 / 2}, h \nu \sim 380 \mathrm{eV}\right)$, we can detect all of the Hf $5 d$ wave functions whether the $d$-electron state is localized near the vacancy or hybridized with the surrounding oxygen atoms (see Fig. 1). Although the obtained spectra become complex due to the on-site core-hole effect, this measurement directly shows the $d$ occupation at the Hf site. 


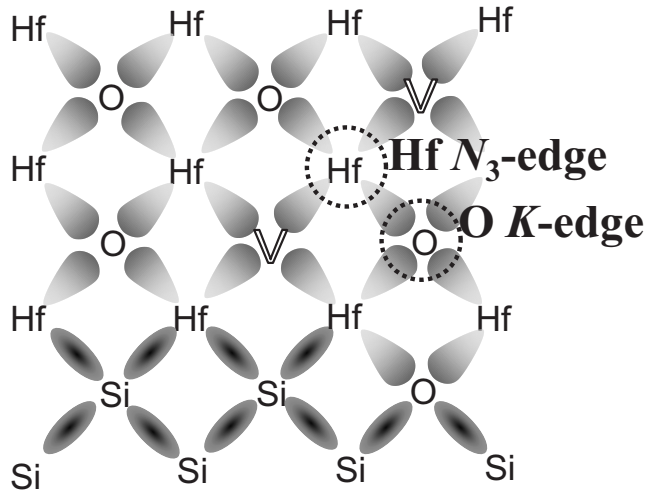

FIG. 1. Schematic diagram of the atomic distribution of $\mathrm{HfO}_{x<2} / \mathrm{Si}$. For simplicity, all the bonds are shown as fourfold. Lobes indicate the intersite wave-function overlaps. The shape and darkness in each lobe represent the electron density. The dashed circles indicate the spatial extent to which each XAS spectrum could detect the electronic structure. In the O $K$-edge XAS, it is impossible to observe the localized Hf $d$-electron state near the oxygen vacancy (denoted as $V$ ), if it were not for the delocalization of the wave functions, in contrast to the $\mathrm{Hf} \mathrm{N}_{3}$-edge XAS.

\section{EXPERIMENTAL DETAILS}

The $\mathrm{O} K$-edge and $\mathrm{Hf} N_{3}$-edge XASs of the oxygendeficient Hf oxide were performed at the 2A EPU6 beamline of the Pohang Light Source (PLS). In order to determine the existence of a long-range FM spin order, we also performed magnetic circular dichroism (MCD) measurements. The MCD spectrum is the difference between two XAS spectra measured using external magnetic fields of mutually opposite directions. Here, a circularly polarized photon should be used, since the relative orientation of its chirality with respect to the direction of the spin moment determines the preference of pumped spins. To align the spin direction, a magnetic field exceeding the coercivity field of the system should be applied. The oxygen-deficient film of $\sim 20 \AA$ was fabricated onto a HF-etched $\mathrm{Si}(100)$ substrate with a $3 \mathrm{~Hz}$ pulsed Nd:YAG laser at a substrate temperature of $700{ }^{\circ} \mathrm{C}$. The base pressure of the growth chamber was 1 $\times 10^{-8}$ Torr and no oxygen flow was supplied during the film growth. The oxygen concentration deduced from the intensity ratio of $\mathrm{O} 1 s / \mathrm{Hf} 4 f$ core levels in the in situ X-ray photoemission spectroscopy (XPS) spectra was approximately 1.8 (i.e., $10 \%$ oxygen deficiency). An approximate estimation of the $\mathrm{Hf}$ valence leads to $\sim \mathrm{Hf}^{3.6+}$, i.e., a composite of $60 \%$ of $\mathrm{Hf}^{4+}$ and $40 \% \mathrm{Hf}^{3+}$.

\section{RESULTS AND DISCUSSIONS}

Figure 2 shows the $\mathrm{O} K$-edge XAS spectrum with circularly polarized photons for $\mathrm{HfO}_{\sim 1.8}$. The overall features of the spectrum are similar to those of the previous experiments for $\mathrm{HfO}_{2}$ (Refs. 18-20) as well as the theoretical band calculations for the unoccupied states. ${ }^{10,21-23}$ The spectrum was analyzed using a model cluster calculation with full atomic multiplets, ${ }^{24}$ assuming the composite $\left[d^{0}(60 \%) \oplus d^{1}(40 \%)\right]$ in a perfect monoclinic crystal structure. As already noted,

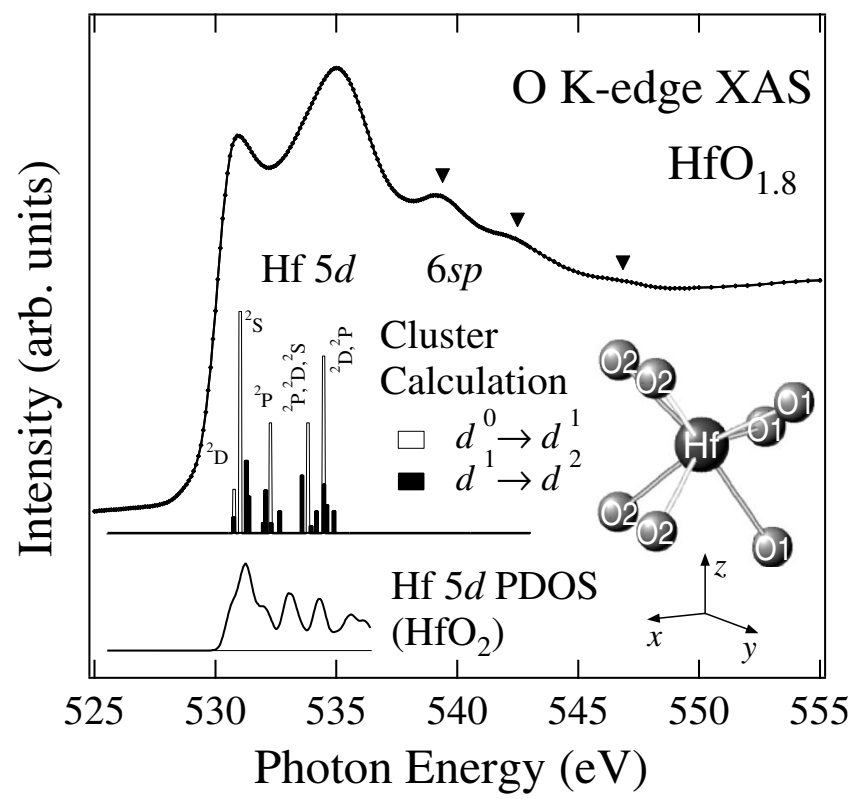

FIG. 2. Unoccupied DOS of $\mathrm{HfO}_{\sim 1.8}$ probed by O $K$-edge XAS with circularly polarized photons. The results of the atomic single cluster model calculations $\left[d^{0}\left(d^{1}\right) \rightarrow d^{1}\left(d^{2}\right)\right]$ are also shown as empty (filled) bars. The cluster considered in this study is shown in the inset. For comparison, the $\mathrm{Hf} 5 d$ PDOS of $\mathrm{HfO}_{2}$ taken from Ref. 1 is shown at the bottom of the figure.

XAS is relevant to the interactions between the near atoms only; therefore, the single cluster model with an Hf atom and its nearest oxygen atoms is sufficient to simulate the XAS spectra. Though the Hf oxide film is in an amorphous phase, the local bonds can be assumed to be similar to those of the bulk monoclinic structure. ${ }^{25}$ The crystal field (CF) split energy ratios were calculated based on the bulk crystal structure, and the overall CF splitting energy was used as a variable scaling parameter to fit the experimental XAS spectra. The one-electron energy level for Hf $5 d^{0} \rightarrow 5 d^{1}$ (empty bars in Fig. 2) is analyzed to be composed of five nondegenerate states: ${ }^{2} D\left(x y / x^{2}-y^{2}\right),{ }^{2} S\left(3 z^{2}-r^{2}\right),{ }^{2} P(x z / y z)$, and two mixed states in the increasing order of energy. (Here the coordinate is taken as in Fig. 2.) The intensities of the five $d$ levels are multiplied by the Hf $5 d-\mathrm{O} 2 p$ hybridization strength using the Harrison's rule (exponent -7 for different interatomic distances $)^{26}$ for the local structure as shown in the figure. The hybridization strength was found to exhibit an anisotropy; the in-planar ${ }^{2} D$ state has much weaker hybridization strength than the other levels $\left(\sim 20 \%\right.$ of ${ }^{2} S$ and $\left.{ }^{2} P\right)$. The $5 d^{1} \rightarrow 5 d^{2}$ simulation (filled bars) shows the similar results, except an overall broadening of features due to the atomic multiplets in the $d^{2}$ final state. ${ }^{27}$ The spectra simulated with the overall CF energy of approximately $4 \mathrm{eV}$, agree with the experimental spectrum and with the calculated Hf $5 d$ PDOS (Ref. 1) shown in Fig. 1.

A slight difference in the XAS spectra of the oxygendeficient film from the stoichiometric film can be seen in the features near the band gap as shown in Fig. 3. For comparison, we appended the XAS spectrum of the stoichiometric $\mathrm{HfO}_{2}$ film measured under the same conditions. Both spectra were normalized approximately preserving the total absorp- 


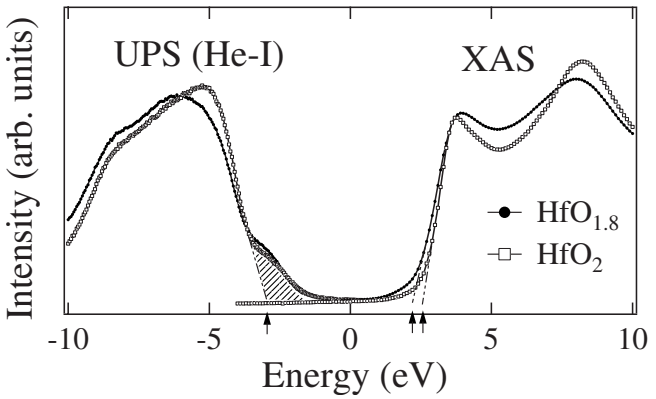

FIG. 3. Combined (normalized) UPS and XAS spectra of $\mathrm{HfO}_{\sim 1.8}$ and stoichiometric $\mathrm{HfO}_{2}$, for DOS near the band gap. The UPS spectrum for $\mathrm{HfO}_{1.8}$ is taken from Ref. 32. Here, the position of the chemical potentials (zero energy in the figure) for both UPS and XAS spectra were arbitrarily chosen to be approximately in the middle of the band gap, preserving the band gap of bulk $\mathrm{HfO}_{2}$ $(\sim 5.7 \mathrm{eV})$. Dotted lines show the extrapolations to obtain the approximate energy positions (arrows) of the $\mathrm{CB}$ and VB edges, and the shaded area indicates the contributions of the Si substrate or the interface state.

tion intensity. The spectrum of oxygen-deficient film appears broader than that of the stoichiometric one. We checked the broadness for many measurement points on each sample and found the consistency for each sample. Thus, the broadness can be regarded as an intrinsic property of the oxygendeficient film. Though no prominent gap state is observed in the XAS spectrum of the oxygen-deficient film, the CB edge extends toward the band gap as compared to the stoichiometric one. This can be interpreted as the partial delocalization of the defect state; that is, the defect state might be rather dispersed so that it can also be detected at the oxygen site. Thus, the role of the oxygen vacancy can be regarded as donating electrons back to the Hf site and consequently to the $\mathrm{O}$ site through the $\mathrm{Hf}-\mathrm{O}$ hybridization. In particular, when the vacancy is at the $\mathrm{HfO}_{2} / \mathrm{Si}$ interface, it is easily substituted by the $\mathrm{Si}$ atom to form an O-Hf-Si bonding. This could also slightly enhance the Hf $d$ occupation, although its amount is reduced due to the electron transfer to the $\mathrm{Si}$ site. ${ }^{28}$ However, the CB tail cannot be attributed to the formation of a direct $\mathrm{O}-\mathrm{Si}$ bonding. This is because the band gap of $\mathrm{SiO}_{2}$ $(\sim 9 \mathrm{eV})$ is much larger than that of $\mathrm{HfO}_{2}(\sim 5.7 \mathrm{eV})$; therefore, no structure can exist near the band gap. We can expect that the band gaps of any type of their intermediate state, i.e., the silicate $\left(\mathrm{Hf}_{x} \mathrm{Si}_{y} \mathrm{O}_{4}\right)$, will be between these two values, ${ }^{29,30}$ resulting in the absence of states inside the $\mathrm{HfO}_{2}$ band gap. Therefore, it is evident that the lack of oxygen atom in the oxide or at the interface is responsible for the occurrence of the CB tail state. ${ }^{23}$

If degree of amorphousness in the $\mathrm{Hf}$ oxide is somehow enhanced, this could also extend the $\mathrm{CB}$ edge due to the broadening of the features. ${ }^{31}$ In this case, the tailing should occur in both the VB and $\mathrm{CB}$ edges. In the following paragraph, however, we will show that the tailing does not occur in the VB edge; this manifests that the CB tail is truly caused by the physics of defects, not by the uncertainty in the lattice dynamics. $^{31}$

To verify the existence of the band tails in the occupied
DOS, we also performed ultraviolet photoemission spectroscopy (UPS) using He-I $(h \nu=21.2 \mathrm{eV})$ light source for both the films. (The UPS spectrum for $\mathrm{HfO}_{\sim 1.8}$ is taken from Ref. 32.) The UPS spectra are also normalized with respect to the total intensity. Here, the energy zero is arbitrarily chosen to approximately sustain the energy difference between the lowest XAS edge $(\sim+2.5 \mathrm{eV})$ and the highest UPS main feature $(\lesssim-3 \mathrm{eV})$ to be the known band gap of $\mathrm{HfO}_{2}$ $\left(\sim 5.7 \mathrm{eV}\right.$; Ref. 33). The UPS spectrum of $\mathrm{HfO}_{\sim 1.8}$ film is shifted by $+0.3 \mathrm{eV}$ in order to compensate for the $E_{F}$ shift in the oxide, which was obtained from the cutoff kinetic energies of the secondary electrons in the spectra (not shown here) ${ }^{32}$ this $E_{F}$ shift induces the higher binding energy (BE) shift in the photoemission spectra, because the BE is, by definition, the energy difference between the Fermi level and the core level, while XAS is independent of the $E_{F}$ position because the energy difference between the initial core level and the final conduction level is independent of the Fermi level. The large and broad features around the relative energy of $-8--4 \mathrm{eV}$ correspond to the main $\mathrm{O} 2 p$ occupied state, while the tail toward the band gap (shaded area at approximately $-3 \mathrm{eV}$ in Fig. 3) probably originates from the underlying $\mathrm{Si}$ wafer, ${ }^{34}$ because this VB tail is absent in the UPS spectra of thicker $\left(>100 \AA\right.$ ) $\mathrm{HfO}_{2}$ film (not shown here). A slight increase in the tail in the oxygen deficient film might be a signature of the metallic Hf silicide (Hf-Si bond) at the interface. ${ }^{35}$ However, the VB edge of the oxygen-deficient film does not extend inside the band gap as compared with that of the stoichiometric sample. Though it is difficult to unambiguously analyze these band-tail states in both the occupied and unoccupied DOSs, we can observe that only the unoccupied DOS near the band gap tends to extend inside the band gap in the presence of oxygen vacancy. ${ }^{10,21}$ This result should be correlated with the lifting of Fermi level because the virtual transition to $\mathrm{CB}$ becomes easier.

Up to this point, we have shown experimental evidence that the oxygen vacancy lowers the $\mathrm{CB}$ edge and increases the effective Fermi level. The detailed mechanism explaining how the vacancy formation lowers the $\mathrm{CB}$ edge could be revealed by a further consideration of the atomic rearrangements or the relaxation following the vacancy formation. For example, the disorder in the positions of the vacant sites can play a role in broadening the CB DOS and consequently in reducing the band gap. Further, the energy position of the defect level is found to depend on the atomic arrangements after the vacancy formation. ${ }^{3}$ The reduction in the mean Hf-O bond length $(\sim-0.1 \AA)$ and in the number of bonds, in the presence of oxygen vacancy, ${ }^{32}$ will influence the detailed balance in the charge dynamics between the two atomic species. The CB lowering can also be correlated with the broadening of features influenced by the valence configurations (the number of electrons) of the remaining atoms, as shown in the simulation results in Fig. 2. Further investigation is required to completely understand the mechanism.

Now we check if there is a long-range FM spin order. The Hf $N_{3}$-edge XAS spectra obtained with circularly polarized photons are shown in Fig. 4(a). The external magnetic field was maintained perpendicular to the sample plane with $H$ $= \pm 2500 \mathrm{Oe}$, which is much larger than the reported ferro- 


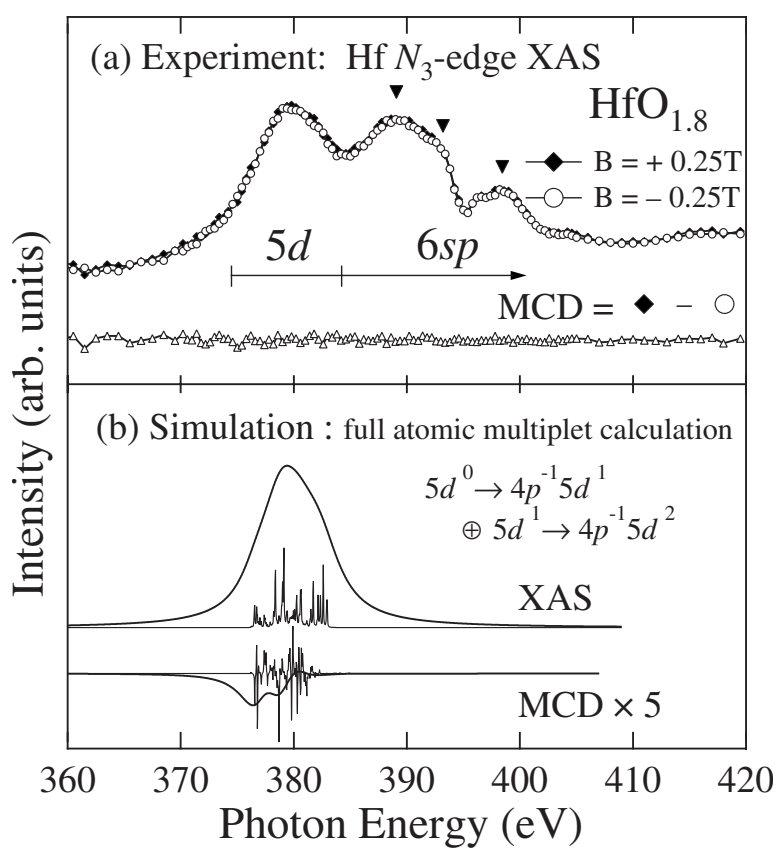

FIG. 4. (a) Hf $N_{3}$-edge XAS with circularly polarized photons. The difference spectrum or MCD has no feature, indicating no longrange FM spin order. (b) $\mathrm{Hf} \mathrm{N}_{3}$-edge XAS/MCD multiplets (spikes) and their broadened spectra obtained by the full atomic cluster model calculations for the composite of $d^{0}(60 \%) \oplus d^{1}(40 \%)$.

magnetic coercivity field of the $\mathrm{HfO}_{2}$ film. ${ }^{11}$ The difference spectrum, i.e., the MCD spectrum exhibits no clear feature and remains zero within the experimental uncertainty. This indicates that there is no $\mathrm{Hf} d$-electron-related FM spin order. One could argue that the density of the oxygen vacancy is too small to detect FM; however, the value of $10 \%$ is larger than the dopant density in conventional diluted magnetic semiconductors (DMS). ${ }^{36}$

To analyze the spectral features, we appended in Fig. 4(b) the simulation result obtained by the full atomic multiplet calculation for the $\mathrm{Hf} N_{3}$-edge XAS $\left(4 p_{3 / 2} \rightarrow 5 d\right)$ with the parameters similar to that of the $\mathrm{O} K$-edge XAS, except for the $4 p-5 d$ Coulomb interactions. ${ }^{37}$ As was the case in the $\mathrm{O}$ $K$-edge XAS simulation, the simulated spectra are obtained by the sum of two transitions, $5 d^{0} \rightarrow 4 p^{5} 5 d^{1}$ and $5 d^{1}$ $\rightarrow 4 p^{5} 5 d^{2}$. The features of the latter are shifted by $U-F_{p d}^{0}=$ $-2 \mathrm{eV}^{37}$ with respect to those of the former. The result of the calculation is in excellent agreement with the experimental spectra; therefore, it provides definite peak assignments; the $\mathrm{Hf} 4 p_{3 / 2} \rightarrow 5 d$ transition is the largest feature, while the other features in the higher photon energies are attributed to the $\mathrm{Hf}$ $4 p_{3 / 2} \rightarrow 6 s p$ transition. Contrary to $2 p \rightarrow 3 d$ transition (as in the case of transition metal ions), the feature of the Hf $4 p$ $\rightarrow 5 d$ transition is not much stronger than $4 p \rightarrow 6 s p$, because of its lower photoabsorption cross sections. The features of Hf $6 s p$ for both the edges are denoted by $\boldsymbol{\nabla}$ in Figs. 2 and 4. The peak positions almost coincide, confirming our peak assignments. The simulated MCD signal for Hf $5 d$ [Fig. 4(b)] is small due to the small anisotropy in the unoccupied electron states $\left(d^{8-9}\right)$. Although the sensitivity of the MCD measurement on magnetism is low, no correlation was observed between the experimental and simulated MCD spectra. This confirms that the small modulation in the experimental spectrum is caused by the noise, not the possible long-range magnetism.

Therefore, we can conclude that there is no preference in spin selection in the electron occupation to either Hf $5 d$ or $6 s p$; this evidently proves "no ferromagnetic spin order" in the case of oxygen vacancy. However, the electron occupation number at an Hf site should be nonzero; otherwise, the electronic configurations of the next nearest $\mathrm{O}$ sites, which was shown in the $\mathrm{O} K$-edge XAS spectra (Fig. 3), should never be influenced by the presence of oxygen vacancy. Thus, the absence of FM spin order can be explained by the absence of long-range correlation between the $d$ electrons in the neighboring Hf sites, rather than the absence of the $d$ electron itself.

\section{CONCLUSION}

We conclude our arguments with two remarks: the oxygen vacancy in the $\mathrm{Hf}$ oxide slightly enhances the CB DOS. However, it does not involve the long-range ferromagnetic spin order in the oxide because of the absence of intersite electron correlation.

\section{ACKNOWLEDGMENTS}

This study is supported by the Korean Science and Engineering Foundation through the Center for Strongly Correlated Materials Research at the Seoul National University, and the experiments at PLS were supported in part by MOST and POSTECH.

\footnotetext{
*Author to whom all correspondence should be addressed. Electronic mail: sjoh@plaza.snu.ac.kr

${ }^{1}$ P. W. Peacock and J. Robertson, J. Appl. Phys. 92, 4712 (2002).

${ }^{2}$ Y.-C. Yeo, T.-J. King, and C. Hu, J. Appl. Phys. 92, 7266 (2002).

${ }^{3}$ K. Xiong, J. Robertson, M. C. Gibson, and S. J. Clark, Appl. Phys. Lett. 87, 183505 (2005).

${ }^{4}$ J. L. Gavartin, D. M. Ramo, A. L. Shluger, G. Bersuker, and B. H. Lee, Appl. Phys. Lett. 89, 082908 (2006).

${ }^{5}$ J. Robertson, K. Xiong, and S. J. Clark, Thin Solid Films 496, 1
}

(2006).

${ }^{6}$ H. Takeuchi, D. Ha, and T.-J. King, J. Vac. Sci. Technol. A 22, 1337 (2004).

${ }^{7}$ A. Kerber, E. Cartier, L. Pantisano, R. Degraeve, T. Kauerauf, Y. Kim, G. Groeseneken, H. E. Maes, and U. Schwalke, IEEE Electron Device Lett. 24, 87 (2003).

${ }^{8}$ J. X. Zheng, G. Ceder, T. Maxisch, W. K. Chim, and W. K. Choi, Phys. Rev. B 75, 104112 (2007).

${ }^{9}$ J. Kang, E.-C. Lee, and K. J. Chang, Appl. Phys. Lett. 84, 3894 
(2004).

${ }^{10}$ E. Cockayne, Phys. Rev. B 75, 094103 (2007).

${ }^{11}$ M. Venkatesan, C. B. Fitzgerald, and J. M. D. Coey, Nature (London) 430, 630 (2004).

${ }^{12}$ J. M. D. Coey, M. Venkatesan, P. Stamenov, C. B. Fitzgerald, and L. S. Dorneles, Phys. Rev. B 72, 024450 (2005).

${ }^{13}$ N. H. Hong, J. Sakai, N. Poirot, and V. Brizé, Phys. Rev. B 73, 132404 (2006).

${ }^{14}$ D. W. Abraham, M. M. Frank, and S. Guha, Appl. Phys. Lett. 87, 252502 (2005).

${ }^{15}$ C. Das Pemmaraju and S. Sanvito, Phys. Rev. Lett. 94, 217205 (2005).

${ }^{16}$ G. Bouzerar and T. Ziman, Phys. Rev. Lett. 96, 207602 (2006).

${ }^{17}$ H. Weng and J. Dong, Phys. Rev. B 73, 132410 (2006).

${ }^{18}$ S. Toyoda, J. Okabayashi, H. Kumigashira, M. Oshima, K. Yamashita, M. Niwa, K. Usuda, and G. L. Liu, J. Appl. Phys. 97, 104507 (2005).

${ }^{19}$ G. Lucovsky, Y. Zhang, G. B. Rayner, Jr., G. Appel, H. Ade, and J. L. Whitten, J. Vac. Sci. Technol. B 20, 1739 (2002).

${ }^{20}$ M.-H. Cho, D. W. Moon, S. A. Park, Y. K. Kim, K. Jeong, S. K. Kang, D.-H. Ko, S. J. Doh, J. H. Lee, and N. I. Lee, Appl. Phys. Lett. 84, 5243 (2004).

${ }^{21}$ A. S. Foster, F. Lopez Gejo, A. L. Shluger, and R. M. Nieminen, Phys. Rev. B 65, 174117 (2002).

${ }^{22}$ A. B. Mukhopadhyay, J. F. Sanz, and C. B. Musgrave, Phys. Rev. B 73, 115330 (2006).

${ }^{23}$ K. Xiong, P. W. Peacock, and J. Robertson, Appl. Phys. Lett. 86, 012904 (2005).

${ }^{24}$ A. Tanaka and T. Jo, J. Phys. Soc. Jpn. 61, 2040 (1992).

${ }^{25}$ R. Ruh and W. R. Corfield, J. Am. Ceram. Soc. 53, 126 (1970).

${ }^{26}$ W. A. Harrison, Electronic Structure and the Properties of Solids (Freeman, San Fransisco, 1980).
${ }^{27}$ Here the effect of charges generated by the oxygen vacancy is neglected, so that the overall CF splitting energy is assumed to be independent of the number of $d$ electrons.

${ }^{28}$ M. S. Joo, B. J. Cho, N. Balasubramanian, and D. L. Kwong, IEEE Electron Device Lett. 25, 716 (2004).

${ }^{29}$ H. Kato, T. Nango, T. Miyagawa, T. Katagiri, K. S. Seol, and Y. Ohki, J. Appl. Phys. 92, 1106 (2002).

${ }^{30}$ Y. Kamimuta, M. Koike, T. Ino, M. Suzuki, M. Koyama, Y. Tsunashima, and A. Nishiyama, Jpn. J. Appl. Phys., Part 1 44, 1301 (2005).

${ }^{31}$ P. W. Anderson, Phys. Rev. 109, 1492 (1957).

${ }^{32}$ D.-Y. Cho, C.-H. Min, J. Kim, S.-J. Oh, and M.-G. Kim, Appl. Phys. Lett. 89, 253510 (2006).

${ }^{33}$ M. Balog, M. Schieber, M. Michman, and S. Patai, Thin Solid Films 41, 247 (1977).

${ }^{34}$ S. Sayan, T. Emge, E. Garfunkel, X. Zhao, L. Wielunski, R. A. Bartynski, D. Vanderbilt, J. S. Suehle, S. Suzer, and M. Banaszak-Holl, J. Appl. Phys. 96, 7485 (2004).

${ }^{35}$ D.-Y. Cho, K.-S. Park, B.-H. Choi, S.-J. Oh, Y. J. Chang, D. H. Kim, T. W. Noh, R. Jung, and J. C. Lee, Appl. Phys. Lett. 86, 041913 (2005).

${ }^{36}$ J. M. D. Coey, M. Venkatesan, and C. B. Fitzgerald, Nat. Mater. 4, 173 (2005).

${ }^{37}$ Here the Slater integrals for $\mathrm{Hf}^{3+}\left(d^{1}\right) / \mathrm{Hf}^{2+}\left(d^{2}\right)$ final states were taken as the reduced values by less than one-half from those calculated for $\mathrm{Hf}^{3+} / \mathrm{Hf}^{2+}$ ion by using Cowan's code for HartreeFock method: for $\operatorname{Hf}^{3+}\left(d^{1}\right), F_{p d}^{2}=9.447, G_{p d}^{1}=2.187$, and $G_{p d}^{3}$ $=2.239 \mathrm{in} \mathrm{eV}$; and for $\operatorname{Hf}^{2+}\left(d^{2}\right), F_{d d}^{2}=13.102, F_{p d}^{4}=9.086, F_{p d}^{2}$ $=9.332, G_{p d}^{1}=2.124$, and $G_{p d}^{3}=2.183$ in eV. Hubbard $U$ and $F_{p d}^{0}$ were set to $2 \mathrm{eV}$ and $4 \mathrm{eV}$, respectively, and the $5 d$ spin-orbit coupling constant was set to about $0.1 \mathrm{eV}$ throughout the calculation. For more details of the calculational method, refer to R. D. Cowan, The Theory of Atomic Structure and Spectra (University of California Press, California, 1981). 